\title{
An observed short-term motor memory effect for isometric force emission
}

\author{
STEPHEN C. FOWLER and JOSEPH M. NOTTERMAN \\ Princeton University, Princeton, New Jersey 08540
}

\begin{abstract}
Contrary to an earlier study, the accuracy of isometric force production is shown to fall under the influence of short-term motor memory, and in a manner quite similar to that previously observed for linear and rotary movement. Four paid male high school seniors pulled on a control stick with one of four designated target force bands. The target ranges were randomly varied in one phase of the experiment, and were the same for a series of consecutive responses in another. A red light came on after each response, and remained on for one of four durations; subjects were instructed not to respond while the light was on. Key variables accounting for the positive findings in the present experiment appear to be the availability of repetition (or massing), and the utilization of appropriate concurrent motor cues which are not supported by correlated visual cues.
\end{abstract}

Short-term motor memory (STMM), or the decreasing ability to match a previously produced standard within a time span usually measured in seconds, has been demonstrated for both linear and rotary movement (Adams \& Dijkstra, 1966; Posner \& Konick, 1966). Interestingly, however, when the motoi task is isometric force emission (i.e., force exerted against an essentially immovable control stick device), investigators have not observed a progressive increase in absolute error (decreased accuracy) of the matching response as a function of increasing short-term retention interval (Pepper \& Herman, 1970). ${ }^{1}$ They tentatively attributed the unexpected discrepancy in findings to differences in "response set" (Pepper \& Herman, 1970, p. 5). This conclusion was inferred from the fact that undershooting (or negative algebraic error) was evidenced in the production of the matching response in the movement studies, while overshooting was manifested in the force experiment.

Comparison of the procedures used in the movement and force research indicates that key methodological distinctions existed, which-independently of (or interacting with) any difference in response characteristics per se-conceivably also could have accounted for the absence of an STMM effect in the Pepper and Herman data. For example: (1) Neither Adams and Dijkstra nor Posner and Konick gave their subjects any concurrent visual feedback during emission of the standard response; a mechanical stop indicated the final distance or angle of the standard movement. The subjects executed

This research was supported by NIMH Grant 18189 . We are grateful to Jeanette $F$. Koftler for assistance in gathering and processing the data. We also thank Scott Replogle for his assistance, and Donald Weitzman for a critical reading. Reprints may be obtained from either co-author. Dr. Fowler is at the Department of Psychology. University of Mississippi. University, Mississippi 38677. both standard and matching (without-stop) movements under circumstances in which they were prevented from seeing either the hand or the apparatus. Pepper and Herman, however, provided concurrent oscilloscopic feedback (excursion of a line trace) for the standard forces. It is possible, therefore, that their subjects came to rely upon visual rather than proprioceptive information during the course of learning to sense the standards, with consequent loss of precision when they had to match the standards using proprioceptive cues alone (see Posner's comnent regarding dominance of "visual information." 1967, p. 104; also Filion, Fowler, \& Notterman, 1969; and Fleishman \& Rich, 1963). (2) Both of the movement experiments contained not only a routine familiarization phase, but-more importantly-subsequent multiple exposure to each of the standard excursions. ${ }^{2}$ The force experiment also had a familiarization phase. However, the subjects did not subsequently experience any of the standard forces more than once in connection with any of the different retention intervals investigated, and these single exposures were necessarily randomly distributed, since a Latin square design was used. Accordingly, it is possible that the disparity between the movement and force data is attributable either to the small amount of experience the subjects had with the respective standard force $\mathrm{x}$ delay combinations, or to the effect of random presentations of these combinations as such, quite independently of limited practice.

One objective of the present experiment was to clarify the foregoing methodological issues. This was accomplished by precluding access to concurrent visual feedback of standard force magnitudes, and by comparing the effects upon matching precision of nonrepetitive (randomly distributed) vs. repetitive (massed) multiple exposures to each of the standards.

A second objective was to eliminate the problem of 
overshoot observed by Pepper and Herman in their force study. As elucidated under Procedure, the strategy followed was that of employing as standards "bands" of forces, target ranges having upper as well as lower limits.

\section{METHOD}

\section{Subjects}

The subjects were four 17-year-old male high school students, who were paid for their services. All the subjects were right-handed.

\section{Apparatus}

The apparatus consisted of three major sets of components: external cueing and reinforcement displays, an isometric lever and force transducer, and a digital computer.

A vertical array of four green signal lamps was centered on the midline of a $19 \times 15 \times 0.5 \mathrm{~cm}$ panel of stiff plastic, which was supported on a table in front of the subject at just about eye level, and at a distance of approximately $61 \mathrm{~cm}$. The lamps were $4.5 \mathrm{~cm}$ apart from each other. From bottom to top, onset of the different lamps designated, in increasing order, the specitic bands of required forces. A red lamp was located in the upper right-hand corner of the visual display, and functioned as a delay indicator. Also on the table, and within the subject's view and hearing, was a digital counter.

A mounting for the isometric lever was bolted to the underside of a laboratory bench serving as a table, the top of which was $84 \mathrm{~cm}$ above the floor. The lever was a stainless steel rod, $1.2 \mathrm{~cm}$ in diam, and protruded through a slot cut into a horizontal fist rest at table level. The rod was pinned through a similar slot $25 \mathrm{~cm}$ below the fist rest. A Revere load cell (Model USP1-.1-A) was used as a force transducer. The sensing element was placed perpendicularly to the rod. $5 \mathrm{~cm}$ below the rest platform

A Digital Equipment Corporation PDP-12-A computer was interfaced with conventional reed relays, and programmed for manipulation of independent variables, reinforcement contingencies. and data collection.

\section{Procedure}

The overall task demanded of the subjects is best conveyed through the instructions, which were as follows:

"In this experiment you will be required to pull with various forces on this rod or control stick.

"Mounted on the panel before you are 5 small colored lights. The vertical column of $\mathbf{4}$ green lights which, as you see, are numbered 1 to 4 will signal which force level you should exert on the rod. The lowest numbered lamp at the bottom of the column designates the lowest target force; lamp 2 the next higher force, and so on with lamp 4 signaling the highest force required. For example. if lamp 1 is on, you should exert a relatively low force always by pulling the rod toward you with your preferred hand. You will find out whether this was the correct force when you completely release the rod. If the force was correct then this counter will register 1 count-you will hear it click. If your force was either too high or too low, the counter will not operate. You should then try again. After every successful response there is a good chance that the target force will change to another value and this will be signaled by the appropriate green light. Each daily session will consist of 4 parts or runs separated by rest periods. After you have made the required number of correct responses per run (which will show on the counter), all 4 lights will go off and remain off. You should then leave the experimental room for a rest period, after which you will be called back to begin the next run.

"There is no emphasis on speed on this task; the emphasis is on accuracy and holding your errors to as few as possible. During each rest period, you will be told what percentage of the previous run's responses was correct. After each response, regardless of whether it is correct or incorrect, the red light at the upper right corner of the panel will come on. This red light signities that a complete response has been made, that is, that you've finished exerting a force, and have released the rod.

"You must not respond while the red light is on; it will be on for different intervals of time."

The following three procedural implications of the instructions are of special importance: (1) Forces were reinforced only if they appeared within a particularly designated band. Previous research has indicated that unless an upper limit is placed upon force emissions. the tendency is for subjects to exert forces which overshoot a criterion, or single target level (cf. Notterman \& Mintz, 1965 , pp. 101-130). This proclivity probably follows from the fact that subjects are never reinforced for producing forces which are below a given target value, and (unless schedules are deliberately imposed) always reinforced for producing forces at or above the criterion. (In the present instance, reinforcement was in the nature of "yes-no" knowledge of results, consisting as it did of a click emanating from a counter, as well as the digital advancement of the counter itself.) (2) Reinforcement did not appear until after the subject had completed his response. In other words, the subject enitted what he deemed to be a force falling within the designated band, then effectively reduced his pull on the manipulandum to zero. If the force was in the correct band, the response would be followed by a click. Based upon the absence of the click, the subject could not infer whether he was "too high" or "too low"; he could only infer that he had made an error. The direction of the error could be ascertained only through retention of the proprioceptive stimuli stenming from the response itself serving as a guide for the next response, and so on, until the designated response was correctly produced. It should be noted that this reinforcement technique (a moditied free operant situation) departs not only from that employed by Pepper and Herman (visual analog of standard forces), but also from that used by Adams and Dijkstra, and Posner and Konick. In the movement studies, the upper limit of each standard response was indicated by a physical stop to the required excursion. (Parenthetically, this possibly accounts for the tendency toward underestimating the matching response, the subject having been reinforced for "cautiously" approaching the invisible position of the stop during production of the standards.) In the present force experiment, the subject had to rely entirely. upon retention of the proprioceptive stimuli accompanying his response, since (apart from the absence of visual information) no upper physical restraint was inposed. (3) Following each response (whether "correct" or "incorrect"), one of four delays was in effect before the next response could be made. The extent of the delay (or retention interval) was indicated by the red lamp. (For zero delay, the light just flashed.) As implied, the retention under consideration was assumed to involve STMM of the proprioceptive stimuli originating as feedback from the previous response.

The four bands and the four delays were selected after fairly extensive pilot work, and with attention being given to the ranges of forces and the retention intervals used in previous studies. The specific delays finally selected were $0,5,10$, and $20 \mathrm{sec}$. The specified bands chosen were as follows: Band 1, 16.67-22.56 N; Band 2. 22.56-28.93 N; Band 3, 28.93-35.80 N; and Band 4, $35.80-43.15 \mathrm{~N}^{3}$ During the course of the pilot work, the subjects became thoroughly familiar with the signals and the nature of the task required of them. Most of their preliminary experience, however, was contined to the zero-delay condition.

The experiment proper involved two phases. During Phase 1, each subject was required to emit a total of 160 correct (or "in band") responses over the course of each of three daily sessions. A session contained four runs, with a $10-\mathrm{min}$ rest between runs. A "run" was specitied as the emission of 10 correct responses at each of the four bands, with the next momentarily required band after any correct response being assigned quasi-randomly. A total of 40 correct responses was made per run. Delay during any given run was maintained at one of the four intervals. However, the order of delay assignment for a given run was quasi-randomly varied, there being the restriction that each of the four delays was represented during a daily session, a different delay being assigned to each of 

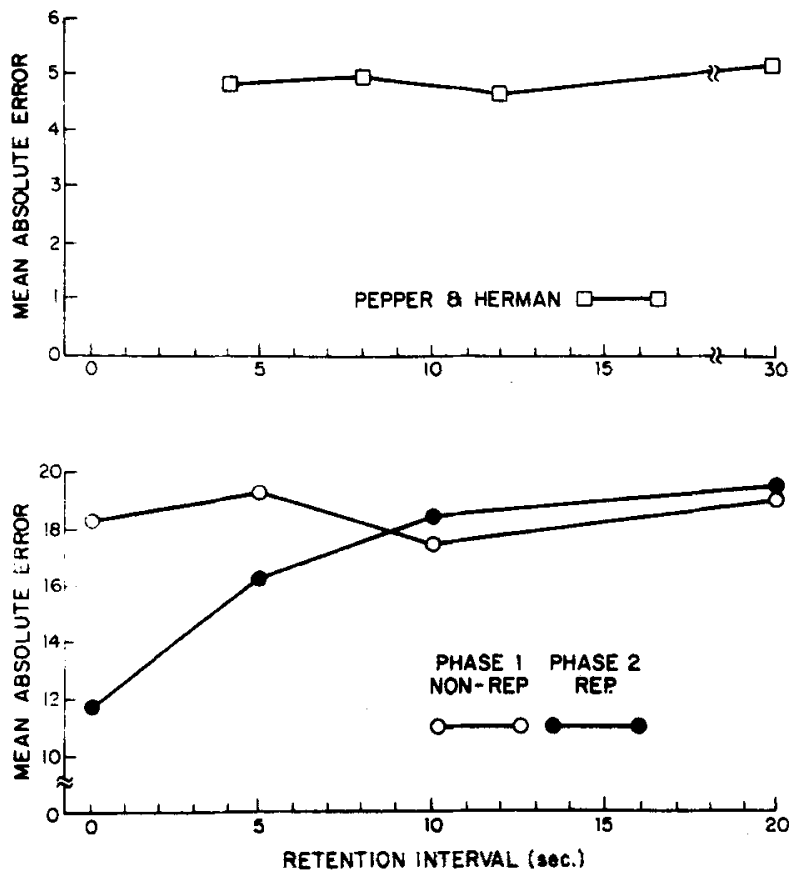

Figure 1. Mean absolute error as a function of retention interval between standard and matching motor responses. For the Pepper and Herman data, absolute error is shown in millimeters, with $1 \mathrm{~mm} \cong 106 \mathrm{~g}$. For the present investigation, absolute error is shown in computer units, with 1 computer unit $=0.2451 \mathrm{~N}$.

the four runs. In other words, by the end of the third session, each of the 10 delay $x$ band combinations had been experienced correctly 30 tines, 10 times (not in succession) during each of the three daily sessions.

During Phase 2 , individual subjects were required to produce a total of 240 correct responses during each of four daily sessions. A daily session consisted of four runs (same rest periods), a run now being specitied as the emission of a total of 60 correct responses in immediate succession, at one of the possible 16 delay $x$ band combinations. The 4 delay $x$ band combinations comprising a daily session of four runs were selected in quasi-random manner, the sole restriction being that none of the 16 combinations could appear more than once during the entire phase. The total number of 60 correct responses per delay $\mathrm{x}$ band combination in Phase 2 (instead of 30 as in Phase 1) was determined on the basis of the pilot work referred to eartier. It was found necessary to double the sum in order to assure that comparable percentages of correct responses were produced for each band at the longest delay interval $(20 \mathrm{sec})$, i.e., where STMM effects were not observed. In this way, the results of the experiment could not be attributed to differences in precision due to irregular appearance of reinforcement, independently of STMM. The subjects (who-as has been noted-were well practiced) reported no differences in exertion or fatigue between the two phases.

\section{RESULTS AND DISCUSSION}

For purposes of comparison with the Pepper and Herman study, performance across all four bands was consolidated for each of the subjects, and is shown in terms of absolute error in the lower half of Figure 1. "Error" was specified as departure from the midpoint of the band in effect at the time each response was made. It appears that repetition of the same delay $x$ band combination (Phase 2) has its STMM advantages up to about $10 \mathrm{sec}$ retention. Beyond that interval, repetition does not seem substantially to affect absolute error.

Table 1 gives, by phase and by subject, absolute error, algebraic error, and percent correct as a function of delay. The grand means of absolute error are the points shown in the bottom part of Figure 1. As indicated in the table, use of the band procedure did, in fact, eliminate any consistent proclivity for overshooting (positive algebraic error) on the part of the individual subjects. Additionally, Table 2 shows that there were no significant differences in algebraic error as a function either of presence or absence of repetition (Phase 1 vs. Phase 2), or of delay interval. The relatively more robust statistical comparisons of percent correct than of absolute error, is probably due

Table 1

Mean Absolute Error, Algebraic Error, and Percentage Correct for Nonrepetitive (Phase 1) and Repetitive (Phase 2) Forces as a Function of Delay Between Responses*

\begin{tabular}{|c|c|c|c|c|c|c|c|c|c|c|c|c|}
\hline \multirow[b]{2}{*}{ Subject } & \multicolumn{3}{|c|}{ 0-Sec Delay } & \multicolumn{3}{|c|}{ 5-Sec Delay } & \multicolumn{3}{|c|}{ 10-Sec Delay } & \multicolumn{3}{|c|}{ 20-Sec Delay } \\
\hline & Abs. & Alg. & Percent & Abs. & Alg. & Percent & Abs. & Alg. & Percent & Abs. & Alg. & Percent \\
\hline $\begin{array}{c}\text { Phase } 1 \\
1 \\
2 \\
3 \\
4\end{array}$ & $\begin{array}{l}21.4 \\
17.3 \\
16.4 \\
17.6\end{array}$ & $\begin{array}{r}6.6 \\
7.6 \\
1.0 \\
.7\end{array}$ & $\begin{array}{l}38.1 \\
48.7 \\
43.5 \\
46.2\end{array}$ & $\begin{array}{l}23.9 \\
14.8 \\
17.0 \\
21.4\end{array}$ & $\begin{array}{r}.4 \\
3.5 \\
8.9 \\
-3.3\end{array}$ & $\begin{array}{l}33.0 \\
55.2 \\
49.0 \\
32.1\end{array}$ & $\begin{array}{l}20.9 \\
17.9 \\
15.4 \\
15.8\end{array}$ & $\begin{array}{r}5.8 \\
9.1 \\
5.5 \\
-5.8\end{array}$ & $\begin{array}{l}32.0 \\
43.4 \\
54.5 \\
48.8\end{array}$ & $\begin{array}{l}22.0 \\
15.2 \\
15.0 \\
24.1\end{array}$ & $\begin{array}{r}9.9 \\
-1.4 \\
.6 \\
-1.6\end{array}$ & $\begin{array}{l}37.9 \\
55.2 \\
51.2 \\
32.3\end{array}$ \\
\hline $\begin{array}{l}\text { Grand } \\
\text { Mean }\end{array}$ & 18.2 & 4.0 & 44.1 & 19.3 & 2.4 & 42.3 & 17.5 & 3.7 & 44.7 & 19.1 & 1.9 & 44.2 \\
\hline $\begin{array}{c}\text { Phase } 2 \\
1 \\
2 \\
3 \\
4\end{array}$ & $\begin{array}{l}12.8 \\
12.2 \\
11.5 \\
10.7\end{array}$ & $\begin{array}{r}-.5 \\
3.2 \\
1.8 \\
-.5\end{array}$ & $\begin{array}{l}62.6 \\
61.9 \\
68.4 \\
74.1\end{array}$ & $\begin{array}{l}15.9 \\
13.5 \\
12.4 \\
23.0\end{array}$ & $\begin{array}{r}2.6 \\
5.8 \\
1.1 \\
11.7\end{array}$ & $\begin{array}{l}53.9 \\
63.2 \\
66.9 \\
41.7\end{array}$ & $\begin{array}{l}19.2 \\
16.7 \\
20.3 \\
17.2\end{array}$ & $\begin{array}{r}2.2 \\
-3.3 \\
.7 \\
-9.5\end{array}$ & $\begin{array}{l}42.6 \\
50.0 \\
42.7 \\
47.8\end{array}$ & $\begin{array}{l}20.2 \\
16.0 \\
22.4 \\
19.5\end{array}$ & $\begin{array}{r}2.6 \\
.1 \\
3.6 \\
-3.5\end{array}$ & $\begin{array}{l}44.4 \\
50.7 \\
38.7 \\
42.7\end{array}$ \\
\hline $\begin{array}{l}\text { Grand } \\
\text { Mean }\end{array}$ & 11.8 & 1.0 & 66.8 & 16.2 & 5.3 & 56.4 & 18.4 & -2.5 & 45.8 & 19.5 & .7 & 44.1 \\
\hline
\end{tabular}

*Absolute and algebraic errors are shown in computer units. The conversion is 1 unit $=.2451 N$. 
Table 2

Analyses of Variance for Absolute Error, Algebraic Error, and Percentage Correct*

\begin{tabular}{llrrr}
\hline & $\begin{array}{l}\text { Source of } \\
\text { Variation }\end{array}$ & df & F & p \\
\hline \multirow{2}{*}{ Absolute } & Repetition & $1 / 21$ & 4.35 & $<.05$ \\
Error & Delay & $3 / 21$ & 3.40 & $<.05$ \\
& Interaction & $3 / 21$ & 2.98 & $<.10$ \\
\multirow{2}{*}{ Algebraic } & Repetition & $1 / 21$ & 1.46 & $>.10$ \\
Error & Delay & $3 / 21$ & .88 & $>.10$ \\
& Interaction & $3 / 21$ & 1.54 & $>.10$ \\
Percentage & Repetition & $1 / 21$ & 13.74 & $<.01$ \\
Correct & Delay & $3 / 21$ & 4.04 & $<.05$ \\
& Interaction & $3 / 21$ & 4.55 & $<.05$ \\
\hline
\end{tabular}

*The analyses are modeled after Type RBF-24 in Kirk (1968).

to the fact that the former dependent variable weights all errors equally, whereas absolute error weights each error in proportion to its departure from band mid point.

Of equal interest in the figure is the comparison of the upper portion with the lower. The Pepper and Herman data are similar to the nonrepetitive band $x$ delay condition (Phase 1) of the present experiment. Because of this similarity. it is evident that the absence of an STMM effect for force cannot be attributed exclusively to "response set." since just one manner of force production was used in both phases of the present study, and yet the results of the two phases are different. It is equally evident that multiple exposures to the standards (or practice) is not responsible for the presence of the STMM effect in the movement studies, or for its absence in the earlier force research, since the current force experiment contains the practice operation in both phases. Therefore. it appears that the most relevant consideration is whether the respective standards succeed each other in repetitive (massed) or nonrepetitive (quasi-random) fashion. By the very fact that the Pepper and Herman experiment contained a Latin square design involving only one exposure per standard, it obviously falls into what is here termed the "nonrepetitive" case. It is currently unknown as to whether their method of force production would have yielded an STMM effect had a repetitive (or massed) procedure been used.

Finally, it must be reiterated that the three previous studies are procedurally different from both Phases 1 and 2 . in that their research involved presentations of standards on a trial-by-trial (or fixed operant) basis. In this study. there was no separate emission of a standard response as such. The proprioceptive feedback stimuli of the previously correct response served as a baseline which was or was not followed by an externally cued demand for approximately the same response. Because of the randomization of band presentations in Phase 1, the previously correct response was generally of a band different from that of the required ensuing response. Accordingly, the effect of reinforcing the prior correct response was not proactive; an analysis of the data failed to reveal any significant effect of a prior successful response on an ensuing response. In Phase 2. the same band was repeated 60 times (at the same delay) before another was designated. Therefore, repetition was effective in Phase 2 . because at the lower retention intervals, feedback stimuli of the previously correct response had not yet become indistinct and the "standard" for band) remained the same.

The foregoing explanation insists that the interresponse times (IRTs) for both phases must fall well within the 10-sec period during which STMM was evidenced. Although IRTs were not obtained directly, a reasonable and conservative approximation is available from rate data computed for all responses (correct and incorrect) emitted during the zero-delay condition. For Phase 1 . the rate was 34.3 responses min: for Phase 2 , it was 37.4 responses min (the difference does not approach signiticance). It follows that IRT was somewhat less than $2 \mathrm{sec}$ for both phases. and that it meets the temporal constraint required by the offered explanation.

By way of conclusion. it should be emphasized that such iniportance as may be attached to the finding that STMM effects are not limited to movements, but occur as well with isometric force production, stems from two sources: (1) A more parsimonious view of STMM is afforded, in that the special topography of the motor response is shown to be less relevant to the demonstration of the phenomenon than is the availability of appropriate motor cues. (2) Perhaps critical for the manifestation of STMM is that these motor stimuli not be supported by correlated exteroceptive (visual) cues. and that the former occur in repetitive (or massed) sequence.

\section{REFERENCES}

Adams. J. A.. \& Dukstra. S. Short-term memory for motor responses. Journal of Experimental Psychology. 1966. 71. $31+318$.

Filion, R. D. L.. Fowler, S. C.. \& Notterman, J. M. Psychophysical evaluation of feedback phenomena as related to precision of force enission: Some methodological considera. tions. American Joumal of Psychology. 1969, 82, 266-271.

Fleishmax. E. A.. \& Rich. S. Role of kinesthetic and spatialvisual abilities in perceptual-motor learning. Joumal of Experimental Psychology, 1963. 66. 6-11.

KIRK. R. E. Experimental design procedures for the behavioral sciences. Belmont. Calif: Wadsworth. 1968. P. 240.

Mintz. D. E.. \& Notrerman. J. M. Force differentiation in human subjects. Psichonomic Science. 1965, 2. 289-290.

votterman. J. M.. \& Mintz. D. E. Dynamics of response Vew York: Wiley. 1965.

Pepper. R. L.. \& Herman. L. M. Decay and interference effects in the short-term retention of a discrete motor act. Journal of Experimental Psychology. 1970, 83 (Monograph Supplement 2).

Posver. M. I. Characteristics of visual and kinesthetic memory 
codes. Journal of Experimental Psychology, 1967, 75, 103-107. PosNer. M. I., \& Konick, A. F. Short-term retention of visual and kinesthetic information. Organizational Behavior and Human Performance, 1966. 1. 71-86.

\section{NOTES}

1. Pepper and Herman described four experiments; the one referred to here is their Experiment I. In Experiment II, Pepper and Herman introduced interpolated activity between force emission and attempted recall, and they obtained evidence for a decrease in absolute error for the single retention interval (20 sec) used. Because Experiment II, and Experiments III and IV as well. primarily examined this "interference" effect (instead of retention interval or amount of practice), only the results of Experintent 1 are immediately germane to the question treated here.

2. Adams and Dijkstra reported on two experiments; the comments in the present paper are based upon their Experiment II. The latter contained three groups, exposed to 1 "reinforcement" (their term for practice or repetition trial) at each particular length, followed by a particular delay; 6 reinforcements prior to a given delay; and 15 reintorcements. respectively. The subjects rested between repetitions. Posner and Konick's subjects (their Experiment II, Rest condition) had four exposures to each angle $x$ delay condition.

3. There was a slight increase in width of band as the bands increased. This was done in order to help compensate for the usually observed increase in variability of force as a function of force magnitude (Mintz \& Notterman, 1965). The range of forces examined was roughly the same as that used by Pepper and Herman-from nearly 0 up to $10 \mathrm{lb}$ maximum.

(Received for publication May 3, 1974; revision received December 4,1974 .) 